\title{
Spatio-temporal patterns of satellite-derived grassland vegetation phenology from 1998 to 2012 in Inner Mongolia, China
}

\author{
SHA Zongyao ${ }^{1,2^{*}}$, ZHONG Jialin ${ }^{1}$, BAI Yongfei ${ }^{3}$, TAN Xicheng ${ }^{1}$, Jonathan LI $^{2}$ \\ ${ }^{1}$ International Software School, Wuhan University, Wuhan 430079, China; \\ ${ }^{2}$ Department of Geography \& Environmental Management, University of Waterloo, Waterloo N2L 3G1, Canada; \\ ${ }^{3}$ Institute of Botany, Chinese Academy of Sciences, Beijing 100093, China
}

\begin{abstract}
Spatio-temporal variations of vegetation phenology, e.g. start of green-up season (SOS) and end of vegetation season (EOS), serve as important indicators of ecosystems. Routinely processed products from remotely sensed imagery, such as the normalized difference vegetation index (NDVI), can be used to map such variations. A remote sensing approach to tracing vegetation phenology was demonstrated here in application to the Inner Mongolia grassland, China. SOS and EOS mapping at regional and vegetation type (meadow steppe, typical steppe, desert steppe and steppe desert) levels using SPOT-VGT NDVI series allows new insights into the grassland ecosystem. The spatial and temporal variability of SOS and EOS during 1998-2012 was highlighted and presented, as were SOS and EOS responses to the monthly climatic fluctuations. Results indicated that SOS and EOS did not exhibit consistent shifts at either regional or vegetation type level; the one exception was the steppe desert, the least productive vegetation cover, which exhibited a progressive earlier SOS and later EOS. Monthly average temperature and precipitation in preseason (February, March and April) imposed most remarkable and negative effects on SOS (except for the non-significant impact of precipitation on that of the meadow steppe), while the climate impact on EOS was found to vary considerably between the vegetation types. Results showed that the spatio-temporal variability of the vegetation phenology of the meadow steppe, typical steppe and desert steppe could be reflected by the monthly thermal and hydrological factors but the progressive earlier SOS and later EOS of the highly degraded steppe desert might be accounted for by non-climate factors only, suggesting that the vegetation growing period in the highly degraded areas of the grassland could be extended possibly by human interventions.
\end{abstract}

Keywords: phenological timing; degradation; harmonic analysis; human activity; climate; restoration

Citation: SHA Zongyao, ZHONG Jialin, BAI Yongfei, TAN Xicheng, Jonathan LI. 2016. Spatio-temporal patterns of satellite-derived grassland vegetation phenology from 1998 to 2012 in Inner Mongolia, China. Journal of Arid Land, 8(3): 462-477. doi: 10.1007/s40333-016-0121-9

Variations in vegetation phenology and their impacts on ecosystem functioning and grassland productivity are becoming serious concerns worldwide, especially in the context of global climate change. Phenology is a biological term that describes recurring natural events. In the case of vegetation, phenology includes bud burst, green-up, flowering, plant maturing and ultimately senescence (Liu et al., 2013). Vegetation phenology plays a fundamental role in regulating photosynthesis and other ecosystem processes and the variation in phenology from vegetative

*Corresponding author: SHA Zongyao (E-mail: zongyaosha@163.com)
Received 2015-07-06; revised 2015-10-24; accepted 2015-11-16
(C) Xinjiang Institute of Ecology and Geography, Chinese Academy of Sciences, Science Press and Springer-Verlag Berlin Heidelberg 2016 
growth to reproductive phase could influence the biomass production of ecosystems (Shinoda et al., 2007; Richardson et al., 2012; Rigge et al., 2013). Phenological events, particularly the date of start of vegetation growing season (SOS) and the date of end of vegetation season (EOS), influence biomass productivity, biodiversity evolution, water balance and carbon balance at local, regional and global scales (Myneni et al., 1997; Liu et al., 2013).

Various factors can influence vegetation phenology (Menzel, 2000; Lambers et al., 2008), among which water and temperature are the most important (Soudani et al., 2012; Liu et al., 2013; Rigge et al., 2013). Piao et al. (2006a) found that the spring green-up of vegetation in the temperate vegetation of China advanced by 0.79 days/a and that the autumn dormancy was delayed by 0.37 days/a during 1982-1999; they further attributed such changes to the warming spring and autumn. Liu et al. (2013) extended the period further to 2006 (i.e. from 1982 to 2006) and stated that the spatial patterns of SOS was delayed in the middle part of Inner Mongolia, China and advanced in the northern and southern parts of the area, presenting significant spatial variations in vegetation phenology. Wu and Liu (2013) assessed the phenological responses (from 1982 to 2006) to changing climate for six temperate biomes of China; their findings showed spatial variations of vegetation phenology among the vegetation biomes as well as advanced spring green-up during the 1980s and early 1990s. Ma and Zhou (2012) observed an earlier spring green-up (during the 1960s-2000s) in China, which they attributed to warming spring, variations in precipitation in spring and other climate phenomena (e.g. Arctic \& North Atlantic Oscillations). Cong et al. (2013) conducted a multi-method investigation over northern temperate China and observed a significant advancing SOS (1.3 \pm 0.6 days per decade) from 1982 to 2010. Recently, Miao et al. (2013) reported no notable earlier green-up over the entire Mongolian Plateau during 1980-2012. Although most of the above studies (in the high altitude region of the Eurasia continent) agree on an advanced green-up or a delayed dormancy, the differences in the observing scale, vegetation type and composition, and contributing factors (e.g. climate variables) lead to individual findings (Jeganathan et al., 2014).

Field observations and remotely sensed time-series data constitute two popular methods of obtaining plant phenological patterns (Myneni et al., 1997; Soudani et al., 2012). Field observations are carried out by biologists and ecologists through botanical inventories, which can record details of phenological events. However, due to the high cost usually associated with field observations, it is difficult to characterize large-scale phenology mapping (Soudani et al., 2012). Remote sensing approach has been successfully used for monitoring vegetation dynamics from regional to global scales (Xie et al., 2008). Furthermore, the remote sensing approach offers the advantage of exploring the driving factors of phenology variations (Piao et al., 2006a; Wang et al., 2010; Shen et al., 2011). Vegetation phenology can be deduced from the annual curves of greenness-sensitive vegetation indices, which are inversely obtained from remote sensing products (Hmimina et al., 2013). The most common index used to derive phenologies is the normalized difference vegetation index (NDVI) (Tucker and Sellers, 1986). So far, the NOAA-AVHRR NDVI, SPOT-VGT NDVI, and MODIS NDVI provide three principal data sources for regional or global vegetation phenology mapping (Tarnavsky et al., 2008). To identify the phenological events, appropriate models or algorithms need to be applied to those NDVI profiles. A range of models (and smoothing algorithms) have been proposed with both pros and cons, including thresholds, derivatives, and model fits (Zhang et al., 2003; Verbesselt et al., 2006; Bradley et al., 2007; Shen et al., 2011; Hmimina et al., 2013; Cao et al., 2015). Besides its advantages in mapping vegetation phenology, a few limitations in remote sensing approach should also be considered. First, the resulting phenological dates from different remote sensing products may be inconsistent (Fensholt et al., 2009; Miao et al., 2013). Inter product comparison must be applied when multiple products are used. Second, the extracted phenological timing may rely on smoothing algorithms (Soudani et al., 2008; White et al., 2009; Atkinson et al., 2012). Lastly, result assessment through the comparison between modeling approaches and field surveys is hard to conduct due to the difficulty in linking field measurements to satellite derived pixel values and the different perspectives in defining vegetation phenology (Wang et al., 2004; White et al., 2009; Hmimina et al., 2013; Pan et al., 2015). 
The Inner Mongolia grassland area is a typical part of the vast grassland region of Eurasia that plays a fundamental part in global carbon cycling (Piao et al., 2008). A few studies have mapped vegetation phenology for Inner Mongolia grassland; however, most studies are limited from coarse-scale representation, e.g. lack of discrimination among vegetation communities (Piao et al., 2006a; Wei et al., 2012; Cong et al., 2013; Liu et al., 2013; Miao et al., 2013). Due to high variations of different vegetation types, the effect of species-wise conditioning should be focused for understanding vegetation phenology (Jeganathan et al., 2014). While many approaches to study the long-term impact of climate changes on vegetation phenology adopt seasonally or annually accumulated or aggregated climate variables (e.g. total precipitation or average temperature in the growing season), recent studies found that the interactions between vegetation dynamics and climate variability should be studied at a smaller temporal scale (e.g. at a monthly scale) (Piao et al., 2006b; Fabricante et al., 2009; Horion et al., 2013). Furthermore, in the recent past, the grassland experienced rapid changes owing to a shift in grassland management strategy driven by widely adopted ecological restoration projects (Mu et al., 2013a). In such a context, the climate fluctuations or human interventions may have imposed impacts on plant phenology (Li and Xie, 2013; Mu et al., 2013a). We thus suspect that the trajectories of the phenological events might have been updated. By applying SPOT-VGT NDVI time series, this study aimed to address three issues of the vegetation phenology in the Inner Mongolia grassland, China: (1) exploring the phenological dynamics at regional and vegetation type scales, (2) comparing the monthly climate impacts on the phenology at vegetation type level, and (3) discussing the underlying causes for progressive phenological shifts, if any.

\section{Study area}

The Inner Mongolia grassland in northern China was selected as the study area (Fig. 1a). As a typical grassland region of the Eurasian continent, it covers an area of $1.2 \times 10^{6} \mathrm{~km}^{2}$, of which nearly $60 \%$ is classified as grassland vegetation. According to the national vegetation map compiled by the Institute of Botany, Chinese Academy of Sciences (IB-CAS), there are 4 main grassland vegetation types dominated by various plant communities: meadow steppe (dominated by Stipa baicalansis and Achnatherum splendens, $13 \%$ of the total area), typical steppe (dominated by Stipa grandis and Leymus chinensis, $28 \%$ of the total area), desert steppe (dominated by Stipa klemenzii, Sheep fescue and Seriphidium gracilescens, $12 \%$ of the total area) and steppe desert (dominated by S. sareptana and S. glareosa, 6\% of the total area). The other area (41\%), including barren lands (sand and urban area), crop lands, water bodies and forests, were excluded (Fig. 1b). The IB-CAS land cover is in accordance with the one from IGBP (grey area in Fig. 1b; Pokrovsky et al., 2003). To counterpart vegetation degradation, the Chinese government have introduced vegetation restoration programs (e.g. human eco-migration projects and sand fixing measures) in the past two decades, particularly in severely degraded areas (Mu et al., 2013a).

The vegetation in Inner Mongolia grassland usually turns green (vegetation green-up) in the middle of April and reaches maturity from early July to end August. In September and October, the vegetation gradually yellows and withers (vegetation senescence). Therefore, the lifecycle of grassland vegetation is as follows: preseason, including February, March and April; growing season, including May, June, July and August; postseason, including September and October; and dormancy season, including November, December, and January.

As Fig. 2a shows, the average temperature in the growing season is $12.1-25.2^{\circ} \mathrm{C}$, and the annual precipitation varies from a negligible amount to nearly $570 \mathrm{~mm}$, most of which is allocated in July and August. An arid region exists in the west (annual precipitation $<200 \mathrm{~mm}$ ), a semi-arid region in the middle (annual precipitation in the range of 200-400 mm), and a semi-humid region in the east (annual precipitation in the range of 400-600 mm) (Fig. 2b). Of the four vegetation types, the meadow steppe is mainly located in the eastern part of the study region (semi-humid with low temperature). The desert steppe and steppe desert are most degraded and mainly distributed in the west (arid region with very little precipitation). The typical steppe covers nearly half of the vegetated area and is located in the eastern and middle part. 

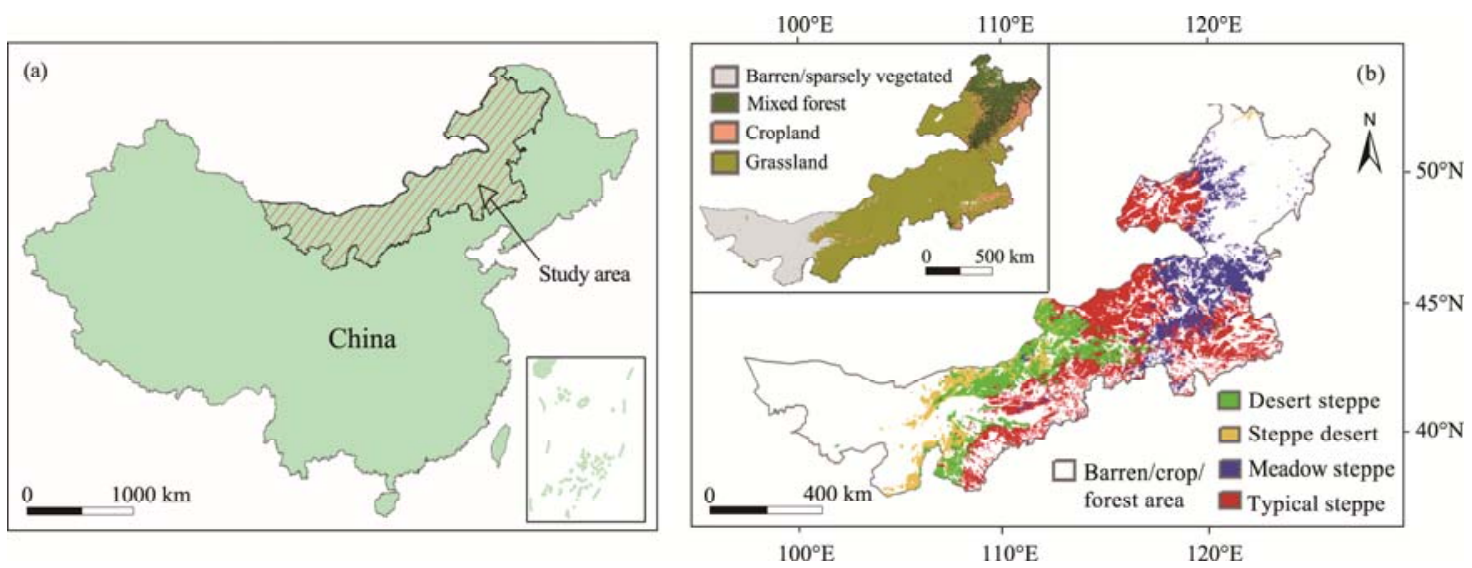

Fig. 1 The study area (a) and vegetation communities in Inner Mongolia (the upper left area in (b) shows the land cover map in Inner Mongolia. Data source: IGBP, from NASA's earth observing system (https://earthdata.nasa.gov))
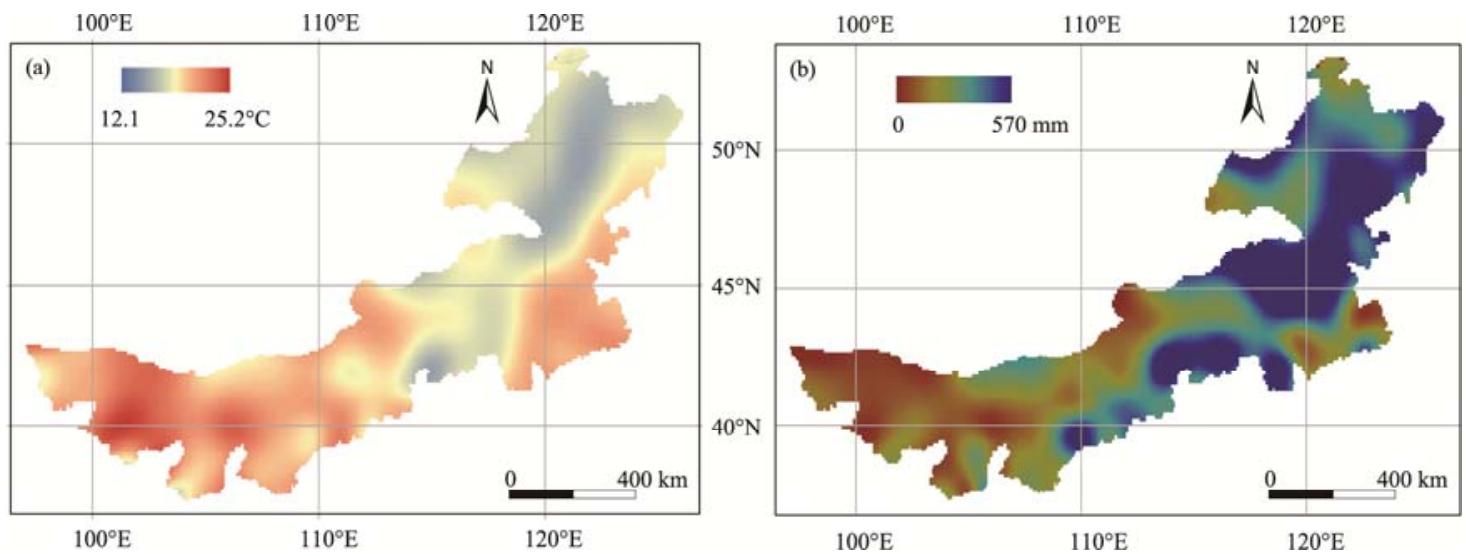

Fig. 2 Average growing season temperature (a) and annual precipitation (b) during 1998-2012 (mapped from data provided by the National Meteorological Bureau of China)

\section{Methodology}

Three phases, i.e. data preparation, data processing and result analysis, are involved in the study (Fig. 3). In Phase I, several steps were taken to prepare qualified dataset. The dataset is listed in Table 1. Ten-day NDVI composites (S10) from the SPOT-VGT were acquired over the study area from April 1998 to December 2012. The band of status map was used to mask bad pixels in the NDVI band. A preliminary data check was then performed to exclude other "invalid" pixels based on a modified version of the criteria from Ding et al. (2013): a mean NDVI from April to September smaller than 0.10; an annual maximum NDVI smaller than 0.20; a maximum NDVI in a month other than between July and September; and a mean NDVI in winter (December, January and February) greater than 0.40 . To minimize the impact from anthropogenically-induced factors to easily accessed areas, road and built-up residential areas from land cover were excluded and a 2-km buffered area surrounding the road and built-up, which is the most influential region by human activities (e.g. grazing), was cut out. The rest pixels were regarded as vegetated area having limited impact from grazing or urbanization. The meteorological data (including monthly average temperature and total precipitation) were collected from 680 weather stations located nationwide (http://cdc.cma.gov.cn). This dataset is the only official meteorological source for the region and has been widely applied in environmental and ecological studies. 


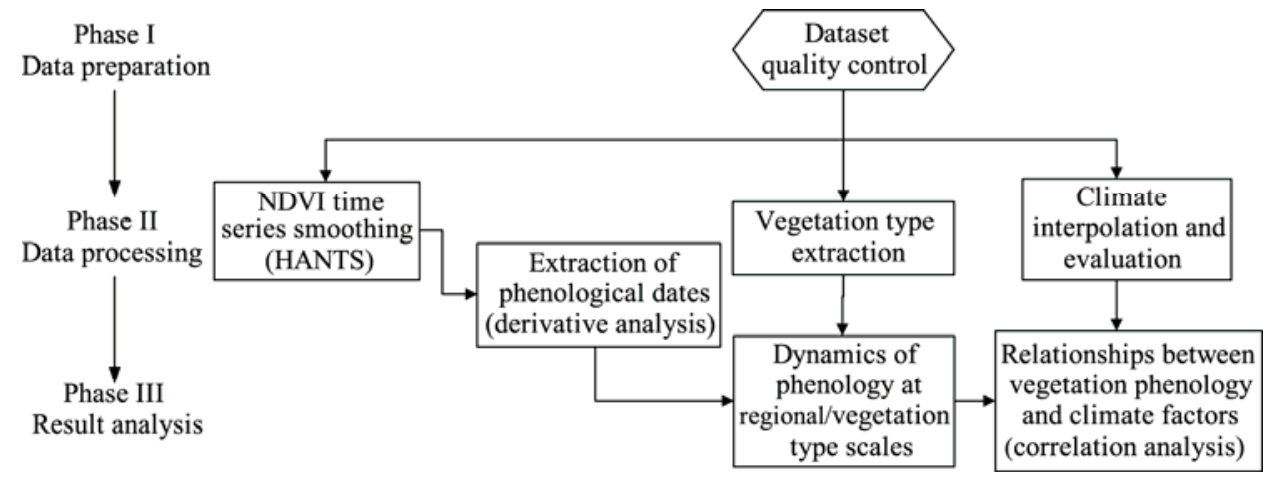

Fig. 3 The framework used in the study

Extraction of phenological dates, area aggregation of vegetation types, and spatial interpolation of meteorological data were processed in Phase II. To compute the phenological dates, NDVI time series smoothing followed by derivative analysis was adopted (Figs. 4a-d). Harmonic analysis algorithm called HANTS (harmonic analysis of NDVI time series) was adopted to smooth the NDVI for each valid pixel within each year. This step built a smoothed annual NDVI curve at a daily interval by fitting the 36 temporal points from the ten-day NDVI synthesis data for each pixel (Fig. 4e). HANTS decomposes a time-dependent periodic phenomenon into a series of constituent sinusoidal functions in which each is defined by a unique amplitude and phase value (Jakubauskas et al., 2002). HANTS is capable of suppressing pronounced outliers or rejecting extreme local minimums and the rebuilt time-series data could successfully reflect the true NDVI profile (Roerink et al., 2000; Julien and Sobrino, 2010). Five parameters were defined in the HANTS analysis: the number of frequencies, a high/low suppression flag, a valid data range, the fit error tolerance, and the degree of over-determinedness (Roerink et al., 2000). In the current study, the number of frequencies was set to 4 . The high/low suppression flag was set to low, intending to avoid local minimums caused by extreme environmental constrains. The valid data range was from 0 to 0.8 . The fit error tolerance was set to 0.04 , and the degree of over-determinedness was set to 13 . Then $1^{\text {st }}$ derivative analysis was applied to extract the phenological timing of both SOS and EOS (Fig. 4f). SOS from remote sensing perspective is defined as the date (in Julian days of year or DOY) that the NDVI reaches the maximum curvature during the spring growth after photosynthetic senescence (Zhang et al., 2003) and EOS is defined similarly as SOS but in a reverse manner. The following prior knowledge helped us determine the phenological dates: the peak NDVI time is reached at the end of July to the middle of August; SOS occurs between late March and early June (gray area A in Fig. 4f); EOS happens in early September and early November (gray area B in Fig. 4f). SOS was computed as DOY reaching the highest derivative value (i.e. difference in NDVI between two consecutive days) with at least five consecutive days having positive derivative during the growing period in spring; EOS was the DOY at the end of senescence obeying similar criterion but in reverse (Fig. 4f). Area aggregation of the four vegetation types was done through overlaying the vegetation cover with the valid pixels.

The climate data were spatially interpolated to produce maps of the monthly average temperature and monthly precipitation with the same resolution as that of the SPOT-VGT NDVI using the thin-plate smoothing splines (ANUSPLIN) approach (Price et al., 2000). Digital elevation model (DEM) was used during the interpolation process (Price et al., 2000; Hong et al., 2005). Error analysis of the interpolated result was conducted by a comparison with the actual measurements using 53 meteorological stations located within the study region; the error reflected by the root mean square error (RMSE) was limited (RMSE $<1.0^{\circ} \mathrm{C}$ for temperature and $\mathrm{RMSE}<2.5 \mathrm{~mm}$ for precipitation). The climate maps were cropped to the boundary of the study area to produce only the region of interest for further analysis. 
Table 1 Data source used in the study

\begin{tabular}{|c|c|c|c|}
\hline Dataset & Data source & Temporal scale & Spatial scale \\
\hline $\begin{array}{l}\text { SPOT-VGT NDVI (bands of } \\
\text { NDVI and status map) }\end{array}$ & $\begin{array}{l}\text { Vegetation programme, } \\
\text { http://www.spot-vegetation.com }\end{array}$ & $\begin{array}{l}10 \text { days, } \\
\text { from 1998-2012 }\end{array}$ & $1 \mathrm{~km} \times 1 \mathrm{~km}$ \\
\hline Vegetation type cover & Institute of Botany, CAS & 2007 & $\begin{array}{l}\text { Re-sampled to } \\
1 \mathrm{~km} \times 1 \mathrm{~km}\end{array}$ \\
\hline Land cover & $\begin{array}{l}\text { National Land Cover Map, } \\
\text { http://www.geodata.cn }\end{array}$ & 2000 & $1 \mathrm{~km} \times 1 \mathrm{~km}$ \\
\hline $\begin{array}{l}\text { Climate data (temperature } \\
\text { and precipitation) }\end{array}$ & $\begin{array}{l}\text { Monthly climate data, } \\
\text { http: //cdc.cma.gov.cn }\end{array}$ & $\begin{array}{l}\text { Monthly, } \\
\text { from 1998-2012 }\end{array}$ & $\begin{array}{l}\text { Spatially interpolated to } \\
1 \mathrm{~km} \times 1 \mathrm{~km}\end{array}$ \\
\hline DEM & $\begin{array}{l}\text { SRTM30, } \\
\text { http://srtm.usgs.gov }\end{array}$ & 2000 & $\begin{array}{l}30 \mathrm{~m} \times 30 \mathrm{~m} \text {, } \\
\text { re-sampled to } 1 \mathrm{~km} \times 1 \mathrm{~km}\end{array}$ \\
\hline Grassland restoration area & Statistic yearbook & 1998-2012 & Yearly \\
\hline
\end{tabular}
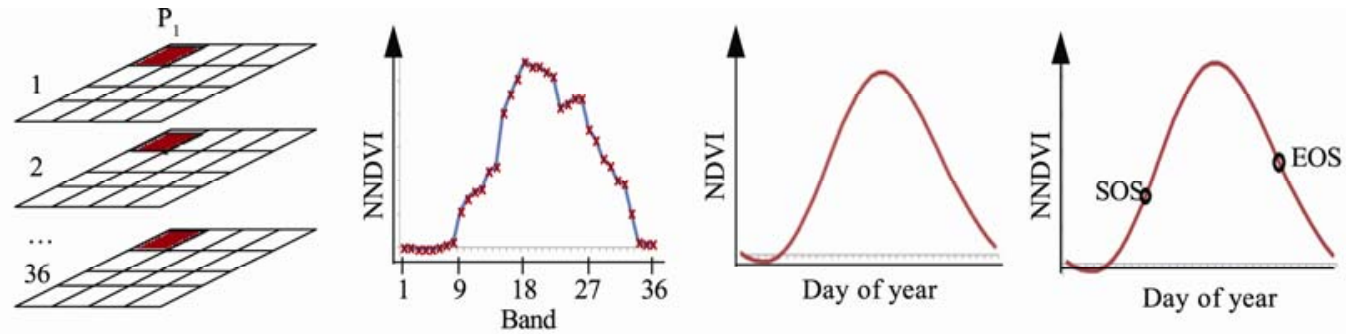

(a) 36-band (NDVI synthesis data)

(b) Annual NDVI dynamics (for pixel $P_{1}$ )

(c) Smoothed annual NDVI

(d) Identified phenological timing

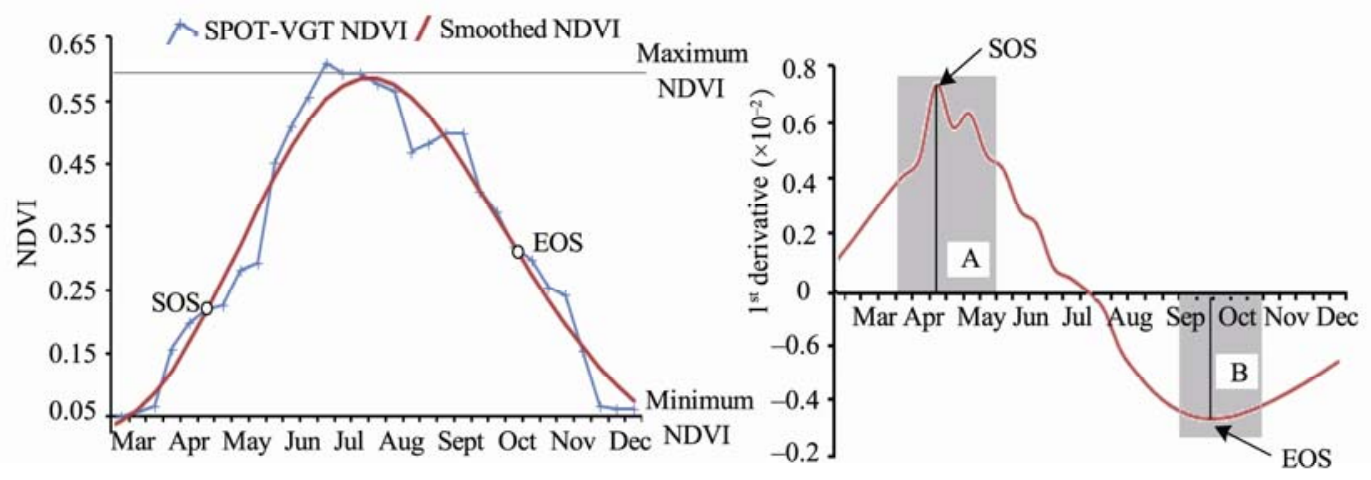

(e) Smoothed NDVI with hollow dots indicating the dates with the most rapid increase and decrease of NDVI

(f) $1^{\text {st }}$ derivative analysis to obtain phenological dates

Fig. 4 Procedures and methods for extracting phenological timing, start of green-up season (SOS) and end of season (EOS), from 10-day SPOT-VGT NDVI synthesis data

We verified that all the variables (SOS, EOS, and the monthly climate) satisfied normal distributions using the S-W (Shapiro-Wilk) test in SPSS (Statistical Package for the Social Sciences). Lastly, the analysis of the spatio-temporal distribution of vegetation phenology and the relationships between the climate variables and the phenology was then performed at regional and vegetation type levels, using univariate statistics, linear regression analysis and correlation analysis in Phase III. At the regional level (i.e. the whole study region), the temporal mean and variation maps of both SOS and EOS during 1998-2012 were analyzed through univariate statistics. In the univariate analysis, the mean value of variable $x$ (SOS or EOS) is given by:

$$
\bar{x}=\frac{1}{n} \sum_{i=1998}^{2012} x_{i},
$$

and the variation of $x$ is defined by the standard deviation estimated by:

$$
s=\sqrt{\frac{1}{n-1} \sum_{i=1998}^{2012}\left(x_{i}-\bar{x}\right)^{2}} .
$$

Where $x_{i}$ is the sample value of the $i^{\text {th }}$ year (from 1998-2012) and $n$ is the sample size (number of 
years).

The spatially averaged SOS and EOS were analyzed against years over the period to map their temporal dynamics using simple linear regression. At the vegetation type level, the SOS and EOS of each vegetation type were compared through univariate statistics. The inter-annual SOS and EOS dynamics of each vegetation type were examined through linear regression analysis. Pearson's correlation coefficient $(R)$ is used for measuring the significance and direction of the linear relationship between the response variable $y$ (SOS or EOS) and a single explanation variable $x$ (year) as:

$$
R_{x y}=\sum_{i=1998}^{2012}\left(x_{i}-\bar{x}\right)\left(y_{i}-\bar{y}\right) / \sqrt{\sum_{i=1998}^{2012}\left(x_{i}-\bar{x}\right)^{2}} \sum_{i=1998}^{2012}\left(y_{i}-\bar{y}\right)^{2} .
$$

Where $x_{i}$ and $y_{i}$ are the $i^{\text {th }}$ paired value of variables $x$ and $y$. Coefficient of determination $\left(R_{x y}{ }^{2}\right)$ is used to indicate the strength of the linear regression function.

The relationships between the climate factors (monthly average temperature and precipitation) and phenological events (SOS and EOS) of each vegetation type were analyzed using correlation analysis. Because SOS occurs mostly at the end of April or early May while EOS usually starts at the end of September or October (Fig. 4e), two different time windows, or the months in a full year cycle (MFYC), were selected in the correlation analysis for SOS and EOS separately. That is, for SOS, MFYC was set as the months from previous May until the current April, and for EOS, MFYC was set as the months from the previous October until the current September. This design is conducive to exploring the possible climate impact of all the months before the phenological events actually occur. The two variables, i.e. the monthly average temperature and precipitation, were found to have significant correlations (result not reported). To reveal the correlation between the phenological timing events ( $y$, including SOS and EOS) and the climate variables ( $x$ or $z$ ) while controlling their interaction effect, Pearson's partial correlation coefficient $(R$.) was applied:

$$
R_{x y \cdot z}=\left(R_{x y}-R_{x z} \times R_{y z}\right) /\left(\sqrt{1-R_{x z}^{2}} \times \sqrt{1-R_{y z}^{2}}\right) .
$$

Where $R_{x y \cdot z}$ is the Pearson's partial correlation coefficient, representing the correlation between variable $x$ and $y$ while eliminating the impact from variable $z$.

\section{Results}

\subsection{General characteristics of the vegetation phenology}

SOS during 1998-2012 varied from 100 to 150 days (average of 127.9 \pm 8.6 days, in mean \pm SD of the DOY), roughly from the middle of April to the end of May (Fig. 5a). The temporal variation of SOS ranged from 3.5 to 30 days (15.5 days on average; Fig. 5b). The EOS dynamics is presented in Figs. 5c and d. EOS started between 250 days and 300 days (average of 274.9 \pm 6.8 days, approximately from the middle of September to the end of October) and exhibited a comparable spatial variation to that of SOS. Nevertheless, the temporal variation showed that, for most of the area, EOS varied within 10 days (average of 7.3 days; Fig. 5d), suggesting that the inter-annual EOS fluctuation was smaller compared with that of SOS (15.5 days).

The spatially averaged SOS and EOS of the study area (regional level) during 1998-2012 are shown in Fig. 6. The result indicates that neither SOS nor EOS presented detectable progressive pattern at the regional level $(P>0.05)$.

\subsection{Spatio-temporal patterns of SOS and EOS at the vegetation type level}

The averaged SOS and EOS varied among the different vegetation types (in mean \pm SD, Fig. 7). The meadow steppe presented an earlier green-up (115.3 \pm 4.6 days) than the other vegetation types, followed by typical steppe, desert steppe and steppe desert $(125.6 \pm 15.9,138.5 \pm 8.5$ and $145.2 \pm 10.9$ days, respectively). The EOS of the different vegetation types had a similar pattern to the SOS (266.0 $\pm 4.4,276.4 \pm 7.2,278.2 \pm 7.3$ and $280.4 \pm 7.0$ days for meadow steppe, typical steppe, desert steppe and steppe desert, respectively). The rankings of both SOS and EOS among the 
(a) Temporally averaged SOS

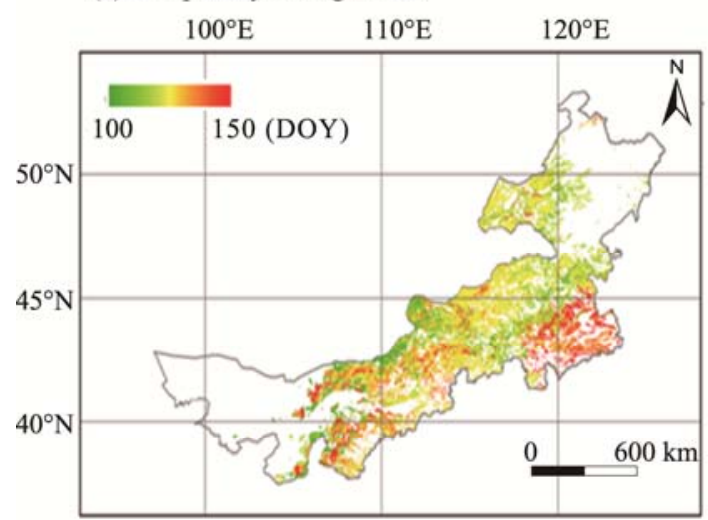

(c) Temporally averaged EOS

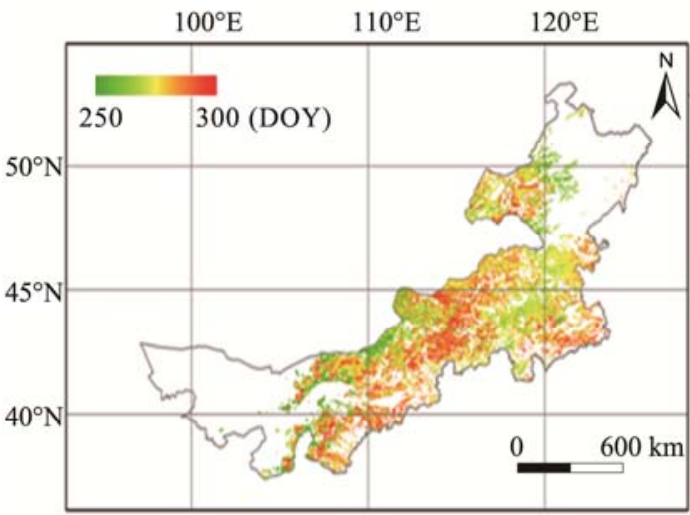

(b) Temporal variation of SOS

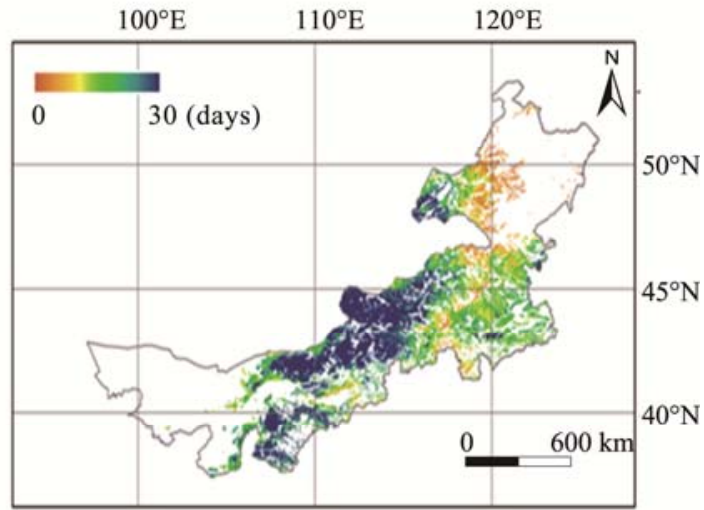

(d) Temporal variation of EOS

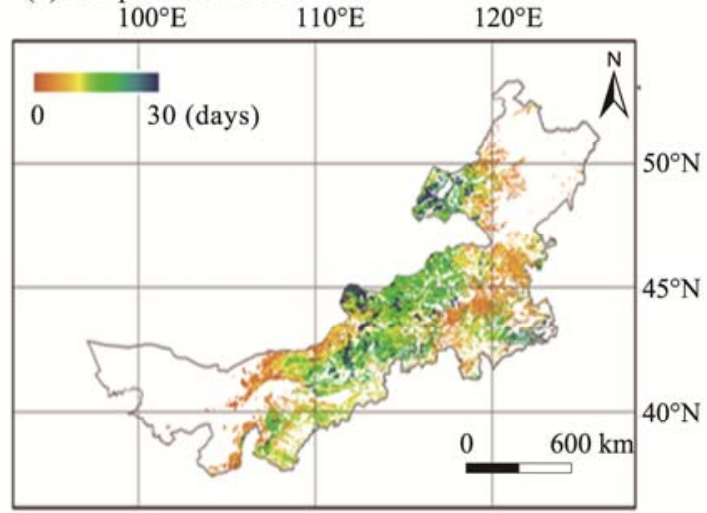

Fig. 5 Temporally averaged SOS (a) and EOS (c) and the corresponding SOS (b) and EOS (d) variations (represented by the standard deviation of SOS and EOS from 1998 to 2012)

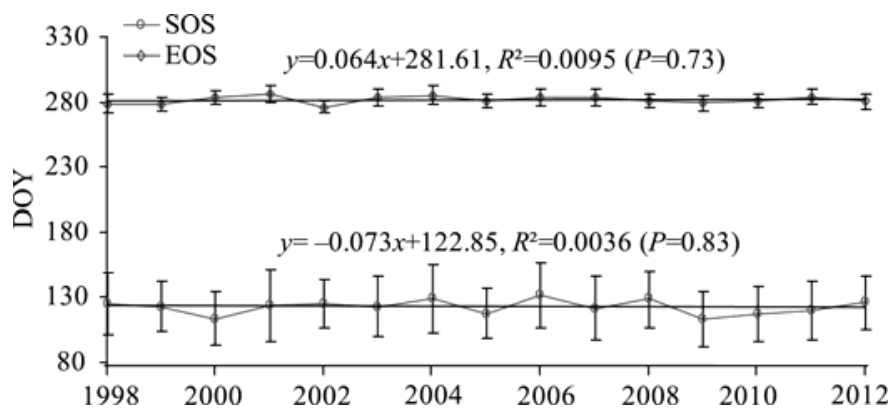

Fig. 6 Temporal trends fitted by linear regression of the spatially averaged SOS and EOS over the study years (from 1998 to 2012) ( $n=15)$. Bar means standard error.

vegetation types are related to the vegetation productivity (based on the NDVI in the growing season, result not shown) as the vegetation productivity is the highest in the meadow steppe, followed by the typical steppe, desert steppe and steppe desert.

The inter-annual changes of SOS and EOS at vegetation type level are shown in Fig. 8. The meadow steppe exhibited a delayed SOS (late green-up) and an advanced EOS (earlier dormancy), with an average rate of 0.51 and 0.54 day/a, respectively, during 1998-2012 (Fig. 8a); however, the shifts were not significant $(P>0.05)$. A similar trend was identified for the typical steppe (Fig. $8 b)$. Both the desert steppe and steppe desert exhibited a delayed EOS; the delay was insignificant for the desert steppe $(P>0.05)$ but significant for the steppe desert (slope $=0.84, R^{2}=0.29, P<0.05$ ). For SOS, no significant change was found for the desert steppe $(P>0.05)$ but significant earlier 
green-up was detected for the steppe desert (slope $=-1.37, R^{2}=0.32, P<0.05$ ). Overall, the inter-annual changes of the phenology exhibited various patterns among the vegetation types; however, unlike the previous findings showing a significant earlier green-up and/or later senescence during the 1980s and 1990s (Ma and Zhou, 2012; Wei et al., 2012), SOS and EOS fluctuations revealed in the current study did not reach a significant level $(P>0.05)$, with the only exception of the steppe desert, which demonstrated a significantly earlier vegetation green-up (1.37 days/a) and later dormancy (0.84 days/a).

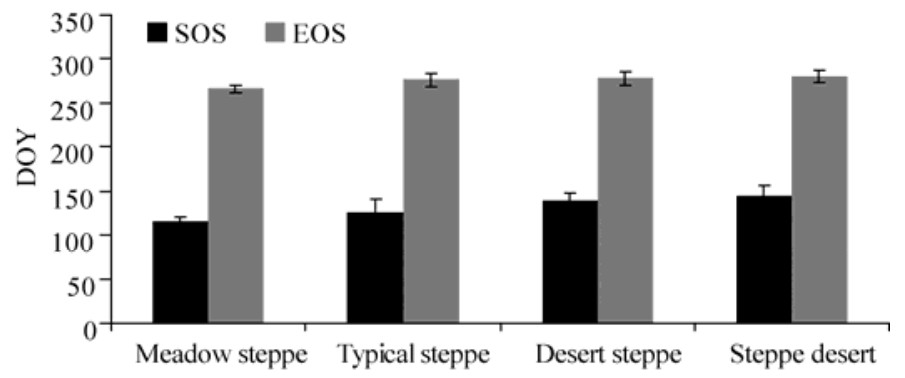

Fig. 7 Spatio-temporally averaged SOS and EOS of different vegetation types with error bars (standard deviations)
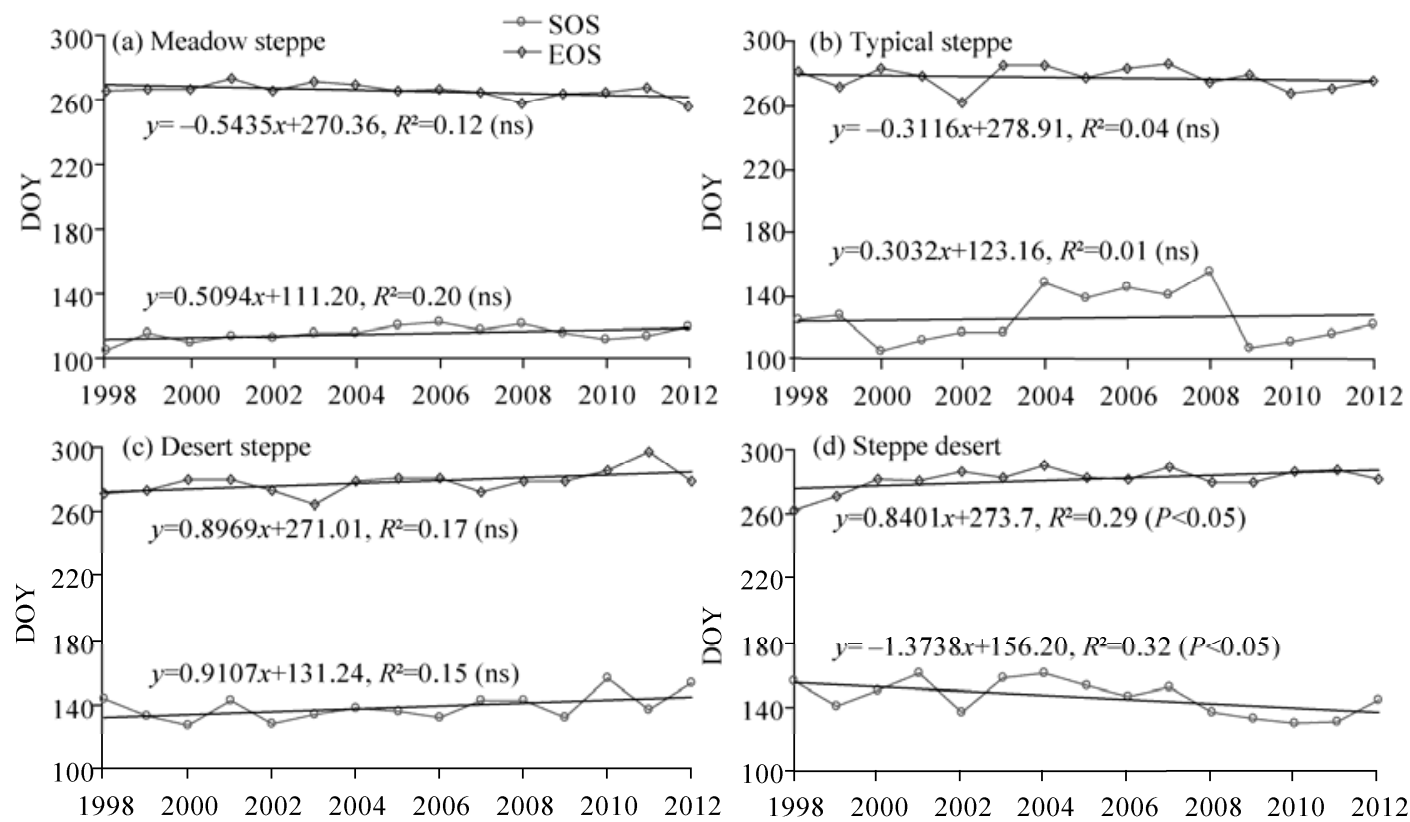

Fig. 8 Inter-annual trend of vegetation phenology (SOS and EOS) for different vegetation types ( $n=15$, non-significant trends are denoted as "ns" and significant trends are accompanied by $P<0.05$ after each regression function)

\subsection{Climate impact on vegetation phenology}

The correlation analysis revealed that the monthly average temperature and total precipitation had important influences on both SOS and EOS, especially of the months in the preseason and postseason (Table 2). Out of the months in a full year cycle (MFYC), SOS was only significantly affected by the climate factors in the preseason months (February, March and April), while EOS could be affected by a much longer period starting from March in the preseason to September in the postseason. The precipitation and average temperature in the early months in MFYC did not have significant effects on either SOS (from the previous May to January) or EOS (from the previous October to February). Nevertheless, the impact varied distinctly among the vegetation types, as detailed below. 
Table 2 Correlation analysis (Pearson's partial correlation coefficient $r, n=15$ ) between the phenological dates (SOS and EOS) of the months in a full year cycle and the climate variables (average monthly temperature and monthly precipitation)

\begin{tabular}{|c|c|c|c|c|c|c|c|c|c|}
\hline & \multirow{2}{*}{ Month $^{\#}$} & \multicolumn{4}{|c|}{ Temperature } & \multicolumn{4}{|c|}{ Precipitation } \\
\hline & & M & $\mathrm{T}$ & D & $\mathrm{S}$ & M & $\mathrm{T}$ & D & $\mathrm{S}$ \\
\hline \multirow[t]{3}{*}{ sos } & February & $-0.58^{*}$ & $-0.62^{*}$ & -0.41 & -0.34 & -0.26 & -0.32 & -0.50 & $-0.55^{*}$ \\
\hline & March & $-0.62^{*}$ & $-0.60^{*}$ & $-0.61^{*}$ & $-0.64^{*}$ & -0.22 & $-0.53^{*}$ & $-0.55^{*}$ & $-0.61^{*}$ \\
\hline & April & $-0.67^{* *}$ & $-0.65^{*}$ & $-0.62^{*}$ & $-0.53^{*}$ & -0.21 & $-0.62^{*}$ & $-0.58^{*}$ & $-0.67^{* *}$ \\
\hline \multirow[t]{7}{*}{ EOS } & March & $0.54^{*}$ & $0.51^{*}$ & 0.21 & 0.13 & 0.21 & 0.38 & 0.30 & 0.22 \\
\hline & April & $0.53^{*}$ & $0.53^{*}$ & 0.24 & 0.20 & $-0.72^{* *}$ & $-0.64^{*}$ & $0.53^{*}$ & $0.54^{*}$ \\
\hline & May & $0.64^{*}$ & $0.53^{*}$ & 0.15 & 0.14 & $-0.64^{*}$ & $-0.54^{*}$ & $0.68^{* *}$ & $0.61^{*}$ \\
\hline & June & 0.14 & -0.11 & $-0.62^{*}$ & $-0.54^{*}$ & $-0.55^{*}$ & $-0.60^{*}$ & $0.67^{*}$ & $0.61^{*}$ \\
\hline & July & -0.18 & -0.22 & $-0.54^{*}$ & $-0.62^{*}$ & 0.22 & 0.23 & 0.36 & $0.56^{*}$ \\
\hline & August & -0.37 & -0.28 & -0.30 & $-0.58^{*}$ & $0.62^{*}$ & $0.53^{*}$ & $0.63^{*}$ & $0.62^{*}$ \\
\hline & September & -0.19 & -0.30 & -0.21 & $-0.66^{*}$ & $0.69^{* *}$ & $0.55^{*}$ & $0.54^{*}$ & $0.64^{*}$ \\
\hline
\end{tabular}

Note: ${ }^{*}$ means significant at $P<0.05$ and ${ }^{* *}$ significant at $P<0.01$. \#, non-significant effect from the previous May to January, is not listed in SOS and non-significant effect from the previous October to February not listed in EOS. M, meadow steppe; T, typical steppe; D, desert steppe; S, steppe desert.

For meadow steppe, the precipitation had no remarkable impact on its SOS, as no significant correlation between the precipitation in any of the MFYC months and SOS was observed $(P>0.05)$. However, the average temperature in February, March and April correlated significantly and negatively to SOS $(P<0.05)$, confirming that the higher temperature in the three months can induce an earlier vegetation green-up. EOS could be affected by both temperature and precipitation. Higher temperature in March, April and May could postpone vegetation dormancy, as evidenced by the significant and positive correlations between EOS and the temperatures $(P<0.05)$. The precipitation in April, May and June exhibited significant and negative correlation with EOS $(P<0.05)$, suggesting that more precipitation in those three months would result in earlier vegetation dormancy. Conversely, more precipitation in August and September had a significant effect in postponing vegetation dormancy.

For typical steppe, the precipitation in March and April may lead to its earlier green-up, as SOS and the precipitation in March and April were significantly and negatively correlated $(P<0.05)$. SOS was found significantly and negatively correlated to the average temperature in February, March and April $(P<0.05)$; thus, the higher temperature in those three months may advance the date of vegetation green-up. In terms of EOS, more precipitation in April, May and June could lead to earlier vegetation dormancy, while higher precipitation in August and September could postpone vegetation dormancy. Delayed dormancy could also be induced by higher monthly temperature in March, April and May.

For desert steppe, the average monthly temperature and precipitation in the preseason, including March and April, exhibited a significant and negative correlation with SOS $(P<0.05)$. Therefore, a warm and rainy preseason is likely to advance SOS. The climate impact on EOS of the desert steppe differs distinctively from the meadow steppe and typical steppe in three aspects. First, most of the monthly precipitation in the preseason and postseason presented significant and positive correlations with EOS $(P<0.05)$, meaning higher precipitation may postpone the vegetation dormancy. Second, the average temperature in June and July had a significant and negative impact on EOS $(P<0.05)$, implying that the higher temperature in June and July would result in earlier vegetation dormancy. Lastly, the average temperature in early stage (March, April and May) did not show significant impact on EOS.

For steppe desert, the temperature in March and April and the precipitation in February, March, and April showed significant and negative effect on its SOS $(P<0.05)$. The monthly precipitation from April to September showed a significant and positive impact on EOS $(P<0.05)$; 
thus, more precipitation in those months meant a delayed growing season. Compared to the other vegetation types, the impact from monthly temperature on the steppe desert was extended to later period (during June and September). The significant and negative correlation between the temperature and EOS suggests that EOS of the steppe desert was more sensitive to temperature changes than other vegetation types, meaning that the higher temperature from June to September would lead to earlier vegetation dormancy.

\section{Discussion}

\subsection{The spatio-temporal characteristics of vegetation phenology}

Significant earlier green-up and delayed vegetation senescence at the Northern Hemisphere middle and high latitudes in the latest few decades were widely recognized (Walther et al., 2002; Jeganathan et al., 2014). For example, previous studies covering an extended area of our study region found an earlier green-up or delayed vegetation senescence (Piao et al., 2006a; Wang et al., 2010; Ma and Zhou, 2012; Cong et al., 2013; Wu and Liu, 2013). Our work examined the grassland vegetation in the Inner Mongolia over the last 15 years (from 1998 to 2012). Our findings detected no significant shifts in the vegetation phenology (except for the steppe desert). The discrepancy between the current study and those from previous ones (earlier green-up and delayed dormancy) may be explained by the differences in the study region and the number of the years (Jeganathan et al., 2014). Specifically, our work focused only on the Inner Mongolia grassland covering four natural vegetation types during 1998-2012, while most other studies focused on a wider area during earlier periods (mainly before 2006). Considering the study grassland is a typical area of the Eurasian continent, we may conclude that the earlier spring green-up and later autumn dormancy observed in the 1980s and 1990s have slowed down or ceased in the new century, although further investigations should be conducted over a broader region.

\subsection{Phenological responses of different vegetation types to climate impacts}

Vegetation phenology is genetically predetermined but can be modulated by environmental conditions (Schaber and Badeck, 2003; Yu et al., 2003). The phenological responses of the four vegetation types could be explained from the interactive effects by the thermal and hydrological gradients (decreasing precipitation from the east to the west and increasing temperature with lower latitudes). More precipitation is likely to advance vegetation green-up (earlier SOS) in drier or warmer regions, while higher temperature can advance SOS in cooler or wetter regions (e.g. Liu et al., 2013; Xu et al., 2013). For example, since the meadow steppe is mainly distributed in relatively cool and wet areas (Figs. 1b, 2a and b), the temperature in all the preseason months showed significant and negative correlation to SOS. Shen et al. (2011) found that soil water could be largely supplemented by thawing of the soil in cooler and wetter regions, which may explain why SOS of the meadow steppe presented no significant correlation to the monthly precipitation.

The EOS fluctuations could also be reflected by the interactions of thermal and hydrological conditions. The positive and significant correlation between the monthly precipitation in August and September and EOS implied that water availability at late stage (before EOS) was essential for prolonging vegetation growth period. Two aggregated areas in the current study could be delineated based on water availability, i.e. relatively wet but cool areas (covered by the meadow steppe and a large part of the typical steppe) and very dry but relatively warm areas (represented by the arid regions covered by the desert steppe and steppe desert). Previous researches have suggested that more water availability had positive effect on the vegetation growth (Liu et al., 2013; $\mathrm{Mu}$ et al., 2013a). The current study confirmed that more precipitation in the vegetation green-up phase (from April to June) could delay EOS in dry and warm areas. However, for the meadow steppe and typical steppe, EOS could be advanced by more precipitation during the vegetation green-up phase, which is probably related to the early poor root development induced by the side effect of more precipitation in the cool and wet areas. Correlation analysis also revealed that higher temperature in March, April and May could significantly delay EOS in the 
wet areas, and EOS could be advanced by higher temperature in later stage in the dry areas (June and July for the desert steppe, and from June to September for the steppe desert). One commonly observed phenomenon in the Northern Hemisphere was the positive effect of higher temperature to the early development of vegetation roots in cool and wet area (Myneni et al., 1997; Liu et al., 2013), suggesting that higher temperature in wet and cool areas may improve vegetation vigor. Our findings confirmed that higher temperature in the early stage (March, April and May) could lead to a delayed EOS in the meadow steppe and typical steppe. In contrast, the higher temperature in late stage (from June to September) would advance EOS in the very dry areas (the desert steppe and steppe desert), especially when accompanied by water shortage (Table 2).

The current work suggests that it is more appropriate to adopt monthly scale than the seasonal or annual scale, though aggregated climatic variables, like accumulated precipitation over the entire growing season, are often adopted in phenology studies (Pokrovsky et al., 2003). Fine temporal scale is believed to be especially important for studying the phenological dynamics of grassland vegetation because, compared to other land covers (e.g. forestry or cropland), grassland vegetation are more sensitive to the climate variations (Wang et al., 2004; Liu et al., 2013). Our study indicated that the climate impact on the vegetation phenology varied considerably between the MFYC months and vegetation types (Table 2). Thus, aggregated variables may be too coarse and unable to detect the monthly detail to the phenological changes.

\subsection{Insight from the progressive phenological shifts of the steppe desert}

Vegetation phenological phases are associated with environmental factors and human activities (Li and Xie, 2013; Mu et al., 2013a). Particularly, the dates of the phenological events for the Inner Mongolia grassland depended on the climatic differences caused by the distance to the southeast coast of the Pacific Ocean (Lee et al., 2002). Non-significant precipitation or temperature updates during 1998-2012 may attribute to the progressive shift at the regional scale or in most vegetation types. However, the phenological dynamics of the steppe desert, which is the least productive and most degraded area, are important to note. The steppe desert only covers a small part (6\%, Fig. 1), but significant advanced SOS by 1.37 days/a and delayed EOS by 0.84 days/a during the study period were observed. This long-term progressive trend (1998-2012) cannot be explained solely by either monthly precipitation or average temperature because no consistent climate trend (e.g. progressively warming spring or autumn) during the study period was observed (results not reported). Although the effect from human activities due to easy accessibility was largely avoided by removing road and built-up resident areas, the phenolgical patterns could still be affected by other factors rather than climate fluctuations. For example, based on the analysis from the statistic yearbook of the region, we found that the national vegetation restoration programs, e.g. the fragile area protection (protecting vegetation by fencing), human eco-migration project, and sand fixing measures were introduced mostly in the degraded regions in the past two decades by the Chinese government ( $\mathrm{Li}$ et al., 2009; Mu et al., 2013b). The implementation of the vegetation restoration projects in those ecologically fragile regions was found to be able to arrest the grassland deterioration (Mu et al., 2013a), and thus the phenological shifts in the steppe desert might be related to those ecosystem improvement programs. For example, the human migration project known as "eco-migration" adopted in Ordors region (an arid grassland area mainly corresponding to the steppe desert and desert steppe) in the Inner Mongolia grassland resulted in significant improvement of vegetable coverage from $30 \%$ in 2000 to $75 \%$ in 2009 (Zhen, 2013). Furthermore, similar ecology restoration projects also contributed significantly to the improved soil conditions (Liu et al., 2013), which can provide positive environment for advancing vegetation green-up and delaying vegetation dormancy. The result suggests that the phenological response of the steppe desert may be more sensitive to human intervention than that of other vegetation types, demonstrating the possibility of prolonging vegetation growing period in the severely degraded areas of the grassland.

\subsection{Limitations of the current study}

Although some important findings were presented, a few limitations should be acknowledged. 
First, the data quality and limited availability of SPOT-VGT NDVI deserve attention in future work. Cloud contamination is still problematic for SPOT-VGT NDVI series even Maximum Value Composite (MVC) was applied in the production process (Vancutsem et al., 2009). The 1 $\mathrm{km} \times 1 \mathrm{~km}$ resolution of SPOT-VGT NDVI also imposes uncertainties, particularly for the steppe desert vegetation due to its low NDVI value; therefore we excluded areas with peak NDVI lower than 0.20, intending to counterpart the low signal-to-noise issue. Besides, the 15-year NDVI series may not be sufficient to predict the inter-annual SOS and EOS trend. Longer time series of remotely sensed images, such as Landsat or AVHRR may provide alternative choices. However, the low data quality of AVHRR could have side effect on modeling results while the higher spatial (but lower temporal) resolution of images from Landsat is challenging to make regional mapping (Xin et al., 2015). Continued land-cover monitoring from SPOT satellites will make improvement for future studies. On the other hand, the methodology used for the vegetation phenology extraction could add further uncertainties. For example, the NDVI smoothing process using HANTS algorithm could inevitably introduce uncertainty to the resulted NDVI curves. The $1^{\text {st }}$ derivative analysis was used to locate SOS and EOS, corresponding to the dates of peak and valley value in the derivative NDVI. However, under certain circumstances (e.g. abnormal temperature fluctuations), multiple peaks or valleys with identical derivative values may make it unreliable in selecting the peak or valley value. As shown in Fig. 4f, there are two peak values (in gray area A); as long as the two peaks were identical in amplitude, uncertainties could be introduced in locating SOS. Furthermore, lack of long-term on-site observation data, the coarse spatiotemporal resolution of NDVI images, and the different nature of satellite derived dates and ground observed phenophases all make it hard to implement systematic verifications through field measurement (Zhang et al., 2003). Instead, our findings are compared to similar studies performed in the same region. The current study indicated that the multi-year average SOS and EOS at regional scale were 127.9 and 274.9 days, respectively; at vegetation type level, the SOSs were 115.3, 125.6, 138.5, and 145.2 days for the meadow steppe, typical steppe, desert steppe and steppe desert, while the corresponding EOSs valued 266.0, 276.4, 278.2, and 280.4, respectively. Using NOAA-AVHRR NDVI in the same area, Lee et al. (2002) examined SOS during 1982-1990 and found that the meadow steppe in eastern part greened up in late April while the typical steppe on the western side greened up mostly by early May. Wu and Liu (2013) reported SOS started averagely at 124-130 days for temperate steppe and temperate desert in northern China during 1982-2006. Piao et al. (2006a) found that SOS was 119-132 in DOY and EOS was about 279-284 during 1982-1999. Last but not least, at current stage an incomplete anthropogenic dataset made it hard to make a detailed analysis on the coupled effect of the climate and human activities to the vegetation phenology.

\section{Conclusions}

Vegetation phenology not only serves as an important signal of climate change and global environment variation but also provides critical information for resources conservation and management. The earlier presence of green land cover and the delay in leaf fall may alter the seasonal climate through the effects of biogeochemical process and physical properties. Information of vegetation phenology dynamics could be used to study real-time foliage development, which is especially important for grazing stock management for the current study region. The SPOT-VGT NDVI was applied to derive the spatio-temporal dynamics of the vegetation phenology during 1998-2012 in the Inner Mongolia grassland in China. The spatio-temporal phenological changes have been demonstrated at regional and vegetation type levels. The monthly climatic influence on the phenological changes of the four vegetation types has been analyzed and compared.

SOS and EOS did not exhibit consistent shifts at either regional or vegetation type level, except for the steppe desert which presented a progressive earlier SOS and later EOS. The monthly average temperature and monthly precipitation affected the phenological timing to different extents and magnitudes among the vegetation types. The temperature and precipitation in the 
preseason months imposed a remarkable and negative impact on SOS (except for the precipitation on SOS of the meadow steppe), and that impact on EOS varied considerably in the MFYC months and directions (positive or negative) among the vegetation types. The spatio-temporal fluctuations of the vegetation phenology could be explained by the interactions of the monthly thermal and hydrological gradients for the meadow steppe, typical steppe, and desert steppe, but the progressive earlier SOS and later EOS of the most severely degraded steppe desert might be attributed to human interventions such as vegetation restoration programs. By analyzing the spatio-temporal patterns of the phenology (SOS and EOS) at vegetation type level and their responses to the monthly climate fluctuations, our work can act as a critical reference for grassland management (e.g. by adjusting stocking rates and season of use). Future work should concentrate on the coupled effects of human activities (e.g. from the vegetation restoration programs) and climate fluctuations on the vegetation phenology.

\section{Acknowledgements}

This study was supported by the Strategic Priority Research Program of the Chinese Academy of Sciences (XDA05050402), the Key Laboratory for Geographic State Monitoring of the National Administration of Surveying, Mapping and Geoinformation (2014-04), and the National Natural Science Foundation of China (41071249, 41371371). The climate data were provided by the National Meteorological Information Center, China Meteorological Administration. We thank the anonymous reviewers for their constructive comments that have helped us to improve the manuscript.

\section{References}

Atkinson P M, Jeganathan C, Dash J, et al. 2012. Inter-comparison of four models for smoothing satellite sensor time-series data to estimate vegetation phenology. Remote Sensing of Environment, 123: 400-417.

Bradley B A, Jacob R W, Hermance J F, et al. 2007. A curve fitting procedure to derive inter-annual phenologies from time series of noisy satellite NDVI data. Remote Sensing of Environment, 106(2): 137-145.

Cao R Y, Chen J, Shen M G, et al. 2015. An improved logistic method for detecting spring vegetation phenology in grasslands from MODIS EVI time-series data. Agricultural and Forest Meteorology, 200: 9-20.

Cong N, Wang T, Nan H J, et al. 2013. Changes in satellite-derived spring vegetation green-up date and its linkage to climate in China from 1982 to 2010: a multimethod analysis. Global Change Biology, 19(3): 881-891.

Ding M J, Zhang Y L, Sun X M, et al. 2013. Spatiotemporal variation in alpine grassland phenology in the Qinghai-Tibetan Plateau from 1999 to 2009. Chinese Science Bulletin, 58(3): 396-405.

Fabricante I, Oesterheld M, Paruelo J M. 2009. Annual and seasonal variation of NDVI explained by current and previous precipitation across Northern Patagonia. Journal of Arid Environments, 73(8): 745-753.

Fensholt R, Rasmussen K, Nielsen $\mathrm{T}$ T, et al. 2009. Evaluation of earth observation based long term vegetation trends-intercomparing NDVI time series trend analysis consistency of Sahel from AVHRR GIMMS, Terra MODIS and SPOT VGT data. Remote Sensing of Environment, 113(9): 1886-1898.

Hmimina G, Dufrêne E, Pontailler J Y, et al. 2013. Evaluation of the potential of MODIS satellite data to predict vegetation phenology in different biomes: an investigation using ground-based NDVI measurements. Remote Sensing of Environment, 132: 145-158.

Hong Y, Nix H A, Hutchinson M F, et al. 2005. Spatial interpolation of monthly mean climate data for China. International Journal of Climatology, 25(10): 1369-1379.

Horion S, Cornet Y, Erpicum M, et al. 2013. Studying interactions between climate variability and vegetation dynamic using a phenology based approach. International Journal of Applied Earth Observation and Geoinformation, 20: 20-32.

Jakubauskas M E, Legates D R, Kastens J H. 2002. Crop identification using harmonic analysis of time-series AVHRR NDVI data. Computers and Electronics in Agriculture, 37(1-3): 127-139.

Jeganathan C, Dash J, Atkinson P M. 2014. Remotely sensed trends in the phenology of northern high latitude terrestrial vegetation, controlling for land cover change and vegetation type. Remote Sensing of Environment, 143: 154-170.

Julien Y, Sobrino J A. 2010. Comparison of cloud-reconstruction methods for time series of composite NDVI data. Remote Sensing of Environment, 114(3): 618-625.

Lambers H, Chapin III F S, Pons T S. 2008. Plant Physiological Ecology ( $2^{\text {nd }}$ ed.). New York: Springer.

Lee R, Yu F, Price K P, et al. 2002. Evaluating vegetation phenological patterns in Inner Mongolia using NDVI time-series analysis. International Journal of Remote Sensing, 23(12): 2505-2512.

Li S, Xie Y C. 2013. Investigating coupled impacts of climate change and socioeconomic transformation on desertification by using multitemporal Landsat images: a case study in central Xilingol, China. IEEE Geoscience and Remote Sensing Letters, 10(5): $1244-1248$. 
Li Y L, Cui J Y, Zhang T H, et al. 2009. Effectiveness of sand-fixing measures on desert land restoration in Kerqin Sandy Land, northern China. Ecological Engineering, 35(1): 118-127.

Liu H, Tian F, Hu H C, et al. 2013. Soil moisture controls on patterns of grass green-up in inner Mongolia: an index based approach. Hydrology and Earth System Sciences, 17(2): 805-815.

Ma T, Zhou C H. 2012. Climate-associated changes in spring plant phenology in China. International Journal of Biometeorology, 56(2): 269-275.

Menzel A. 2000. Trends in phenological phases in Europe between 1951 and 1996. International Journal of Biometeorology, 44(2): 76-81.

Miao L J, Luan Y B, Luo X Z, et al. 2013. Analysis of the phenology in the Mongolian Plateau by inter-comparison of global vegetation datasets. Remote Sensing, 5(10): 5193-5208.

Mu S J, Chen Y Z, Li J L, et al. 2013a. Grassland dynamics in response to climate change and human activities in Inner Mongolia, China between 1985 and 2009. The Rangeland Journal, 35(3): 315-329.

Mu S J, Zhou S X, Chen Y Z, et al. 2013b. Assessing the impact of restoration-induced land conversion and management alternatives on net primary productivity in Inner Mongolian grassland, China. Global and Planetary Change, 108: 29-41.

Myneni R B, Keeling C D, Tucker C J, et al. 1997. Increased plant growth in the northern high latitudes from 1981 to 1991. Nature, 386(6626): 698-702.

Pan Z K, Huang J F, Zhou Q B, et al. 2015. Mapping crop phenology using NDVI time-series derived from HJ-1 A/B data. International Journal of Applied Earth Observation and Geoinformation, 34: 188-197.

Piao S L, Fang J Y, Zhou L M, et al. 2006a. Variations in satellite-derived phenology in China’s temperate vegetation. Global Change Biology, 12(4): 672-685.

Piao S L, Mohammat A, Fang J Y, et al. 2006b. NDVI-based increase in growth of temperate grasslands and its responses to climate changes in China. Global Environmental Change, 16(4): 340-348.

Piao S L, Ciais P, Friedlingstein P, et al. 2008. Net carbon dioxide losses of northern ecosystems in response to autumn warming. Nature, 451(7174): 49-52.

Pokrovsky I, Pokrovsky O, Roujean J L. 2003. Development of an operational procedure to estimate surface albedo from the SEVIRI/MSG observing system by using POLDER BRDF measurements: I. Data quality control and accumulation of information corresponding to the IGBP land cover classes. Remote Sensing of Environment, 87(2-3): 198-214.

Price D T, McKenney D W, Nalder I A, et al. 2000. A comparison of two statistical methods for spatial interpolation of Canadian monthly mean climate data. Agricultural and Forest Meteorology, 101(2-3): 81-94.

Richardson A D, Anderson R S, Arain M A, et al. 2012. Terrestrial biosphere models need better representation of vegetation phenology: results from the North American carbon program site synthesis. Global Change Biology, 18(2): 566-584.

Rigge M, Smart A, Wylie B, et al. 2013. Linking phenology and biomass productivity in South Dakota Mixed-Grass Prairie. Rangeland Ecology \& Management, 66(5): 579-587.

Roerink G J, Menenti M, Verhoef W. 2000. Reconstructing cloudfree NDVI composites using Fourier analysis of time series. International Journal of Remote Sensing, 21(9): 1911-1917.

Schaber J, Badeck F W. 2003. Physiology-based phenology models for forest tree species in Germany. International Journal of Biometeorology, 47(4): 193-201.

Shen M G, Tang Y H, Chen J, et al. 2011. Influences of temperature and precipitation before the growing season on spring phenology in grasslands of the central and eastern Qinghai-Tibetan Plateau. Agricultural and Forest Meteorology, 151(12): 1711-1722.

Shinoda M, Ito S, Nachinshonhor G U, et al. 2007. Phenology of mongolian grasslands and moisture conditions. Journal of the Meteorological Society of Japan, 85(3): 359-367.

Soudani K, Maire G I, Dufrêne E, et al. 2008. Evaluation of the onset of green-up in temperate deciduous broadleaf forests derived from Moderate Resolution Imaging Spectroradiometer (MODIS) data. Remote Sensing of Environment, 112(5): 2643-2655.

Soudani K, Hmimina G, Delpierre N, et al. 2012. Ground-based Network of NDVI measurements for tracking temporal dynamics of canopy structure and vegetation phenology in different biomes. Remote Sensing of Environment, 123: $234-245$.

Tarnavsky E, Garrigues S, Brown M E. 2008. Multiscale geostatistical analysis of AVHRR, SPOT-VGT, and MODIS global NDVI products. Remote Sensing of Environment, 112(2): 535-549.

Tucker C J, Sellers P J. 1986. Satellite remote sensing of primary production. International Journal of Remote Sensing, 7(11): 1395-1416.

Vancutsem C, Pekel J F, Evrard C, et al. 2009. Mapping and characterizing the vegetation types of the Democratic Republic of Congo using SPOT VEGETATION time series. International Journal of Applied Earth Observation and Geoinformation, 11(1): 62-76.

Verbesselt J, Somers B, van Aardt J, et al. 2006. Monitoring herbaceous biomass and water content with SPOT VEGETATION time-series to improve fire risk assessment in savanna ecosystems. Remote Sensing of Environment, 101(3): 399-414.

Walther G R, Post E, Convey P, et al. 2002. Ecological responses to recent climate change. Nature, 416(6879): $389-395$.

Wang Q, Tenhunen J, Dinh N Q, et al. 2004. Similarities in ground-and satellite-based NDVI time series and their relationship to physiological activity of a Scots pine forest in Finland. Remote Sensing of Environment, 93(1-2): 225-237.

Wang X H, Piao S L, Ciais P, et al. 2010. Spring temperature change and its implication in the change of vegetation growth in North America from 1982 to 2006. Proceedings of the National Academy of Sciences of the United States of America, 108(4): 
1240-1245.

Wei H Y, Heilman P, Qi J G, et al. 2012. Assessing phenological change in China from 1982 to 2006 using AVHRR imagery. Frontiers of Earth Science, 6(3): 227-236.

White M A, de Beurs K M, Didan K, et al. 2009. Intercomparison, interpretation, and assessment of spring phenology in North America estimated from remote sensing for 1982-2006. Global Change Biology, 15(10): 2335-2359.

Wu X C, Liu H Y. 2013. Consistent shifts in spring vegetation green-up date across temperate biomes in China, $1982-2006$. Global Change Biology, 19(3): 870-880.

Xie Y C, Sha Z Y, Yu M. 2008. Remote sensing imagery in vegetation mapping: a review. Journal of Plant Ecology, 1(1): 9-23.

Xin Q C, Broich M, Zhu P, et al. 2015. Modeling grassland spring onset across the Western United States using climate variables and MODIS-derived phenology metrics. Remote Sensing of Environment, 161: 63-77.

Xu L, Myneni R B, Chapin III F S, et al. 2013. Temperature and vegetation seasonality diminishment over northern lands. Nature Climate Change, 3(6): 581-586.

Yu F F, Price K P, Ellis J, et al. 2003. Response of seasonal vegetation development to climatic variations in eastern central Asia. Remote Sensing of Environment, 87(1): 42-54.

Zhang X Y, Friedl M A, Schaaf C B, et al. 2003. Monitoring vegetation phenology using MODIS. Remote Sensing of Environment, 84(3): 471-475.

Zhen J Z. 2013. Exploring the impact of eco-migration project, Ordos, Inner Mongolia, China. MSc Thesis. Sweden: Uppsala University. 